\title{
Clinical Characteristics and Outcomes of Primary Breast Lymphoma: The Cleveland Clinic Experience
}

\author{
Tariq Kewan ${ }^{1}$, Fahrettin Covut ${ }^{1}$, Ramsha Ahmed ${ }^{1}$, Abdo Haddad ${ }^{2}$, Hamed Daw ${ }^{2}$ \\ 1. Internal Medicine, Cleveland Clinic - Fairview Hospital, Cleveland, USA 2. Hematology and Oncology, Cleveland \\ Clinic - Fairview Hospital, Cleveland, USA
}

Corresponding author: Tariq Kewan, kewant@ccf.org

\begin{abstract}
Introduction

Primary breast lymphoma (PBL) is a rare malignancy that accounts for less than $0.5 \%$ of all breast malignancies.
\end{abstract}

Materials and Methods

We retrospectively analyzed 36 PBL patients to report the clinical characteristics and outcomes of patients with indolent and aggressive histologic subtypes.

Results

Thirteen (36\%) patients had aggressive and 23 (64\%) had indolent PBL. Marginal zone lymphoma was the most common histologic subtype (33\%). Stage IE, IIE, and IV disease were seen in 27 (75\%), six (17\%), and three (8\%) patients, respectively. Patients with aggressive PBL more often presented with a breast lump and/or B symptoms (unexplained weight loss, fever, night sweats) $(78 \%$ vs. $31 \%, \mathrm{p}=0.005)$. Commonly used treatment modalities for aggressive vs. indolent PBL were chemotherapy alone ( $23 \% \mathrm{vs.} 26 \%, \mathrm{p}=0.8$ ), chemoradiotherapy ( $46 \%$ vs. $9 \%, p=0.009)$, radiotherapy alone ( $15 \%$ vs. $22 \%, p=0.6)$, and observation $(0 \%$ vs. $26 \%, p=0.07$ ), respectively. The five-year overall survival (OS) and progression-free survival (PFS) of PBL patients were 82\% (95\% CI: 67 - 100) and 63\% (95\% CI: 45 - 89), respectively. The five-year OS of patients with aggressive vs. indolent PBL were 92\% (95\% CI: 77 - 100) vs. 80\% (95\% CI: 63 - 100), respectively $(\mathrm{p}=0.6)$. The five-year OS of patients who received $>1,1$, and 0 treatment modalities were $92 \%$ (95\% CI: 77 - 100), 86\% (95\% CI: 63 - 100), and 53\% (95\% CI: 21 - 100), respectively.

\section{Conclusion}

Received 05/07/2020

Review began 05/14/2020 Review ended 05/16/2020 Published 06/14/2020

() Copyright 2020

Kewan et al. This is an open access article distributed under the terms of the Creative Commons Attribution License CC-BY 4.0., which permits unrestricted use, distribution, and reproduction in any medium, provided the original author and source are credited.

In our cohort, the higher utilization of chemoradiotherapy in aggressive PBL was able to overcome the worse prognosis of these patients. At least one treatment modality should be considered in patients with indolent PBL, given that observation alone was associated with a poor prognosis.

Categories: Pathology, Oncology, Hematology

Keywords: primary breast lymphoma, incidence and prognosis, non-hodgkin's lymphoma

\section{Introduction}

Primary breast lymphoma (PBL) is a rare malignancy that accounts for less than $3 \%$ of extranodal lymphoma, $1 \%$ of non-Hodgkin's lymphoma (NHL), and $0.5 \%$ of all breast malignancies [1-3]. PBL was first described in 1972 by Wiseman and Liao as breast tissue infiltration by lymphoma with or without regional lymph node involvement in patients without prior nodal or extranodal lymphoma at the time of diagnosis [4]. This definition was reviewed in 1990 by Hugh et al. [5]. Patients presenting with lymphoma of regional lymph nodes and bilateral breast involvement were also included in the definition of PBL [6]. PBL affects female patients in > 95\% of the cases and involves the right breast more than the left. Diffuse large B-cell lymphoma (DLBCL) is the most commonly reported histological subtype [1-2, 7-9]. Marginal zone lymphoma, follicular lymphoma, and mucosa-associated lymphoma are not as common as DLBCL [1-2, 1011]. In addition, anaplastic large T-cell lymphoma (ALCL) and Hodgkin's lymphoma represent a very small proportion of PBL. ALCL is usually localized and presents as a mass or an effusion. Recently, other PBL subtypes have been reported after plastic surgery procedures [2, 12].

Clinically, PBL can be diagnosed as an incidental finding on mammogram or patients can present with breast swelling and B symptoms (unexplained weight loss, fever, night sweats). Mammographic features of PBL are distinct from invasive breast cancer. The role of breast ultrasound and magnetic resonance imaging 
in PBL diagnosis is not well established yet [7-8, 13]. Retrospective studies reported no benefit of radical mastectomy compared to partial mastectomy or biopsy in terms of overall survival (OS) and progression-free survival (PFS) rates. A combination of chemoradiotherapy has been shown to increase complete remission rates compared to chemotherapy or radiotherapy alone [1, 7, 13-19]. The International Extranodal Lymphoma Study Group reported that a combination of surgery, chemotherapy, and radiation to involvedfield yielded the best prognosis $[9,15,17,19]$.

In view of the rarity of PBL, we hereby report the clinical characteristics and outcomes of PBL patients after different treatment modalities to contribute to defining optimal treatment strategy for this rare lymphoma subtype.

\section{Materials And Methods}

We retrospectively identified patients who were diagnosed with PBL between January 2014 and January 2019 at Cleveland Clinic Foundation. This study was approved by the Institutional Review Board of the Cleveland Clinic (\#18-1303). PBL was defined as breast tissue infiltration by lymphoma with or without regional lymph node involvement in patients without prior nodal or extranodal lymphoma at the time of diagnosis [4]. We included patients with Stage IE, IIE, and IV disease and considered patients with bilateral breast involvement to have Stage IIE PBL [1-2, 5, 9]. The World Health Organization histologic classification was used for histologic classification of PBL [20]. Clinical variables, including age at diagnosis, presenting symptoms, performance status at diagnosis, history of breast implant, affected breast, lymphoma histologic type, stage at diagnosis, treatment modalities (surgery, radiotherapy, chemotherapy, central nervous system chemoprophylaxis), and relapse data, were collected. Aggressive PBL was defined as DLBCL, breast implantassociated ALCL, and unclassifiable B-cell lymphoma with features intermediate between DLBCL and Burkitt's lymphoma, while other histological subtypes were defined as indolent PBL.

Baseline characteristics were compared between the indolent and aggressive PBL groups using a two-sample T-test for continuous variables, Chi-square test, and Fisher's exact test for categorical variables. OS and PFS were estimated by the Kaplan-Meier method and compared by the log-rank test. PFS was calculated from the date of diagnosis to the date of first recurrence, progressions, or death. A p-value of less than 0.05 was considered statistically significant. All statistical calculations were made using R statistical software version 3.4.0 (R Foundation for Statistical Computing, Vienna, Austria).

\section{Results}

A total of 36 patients with PBL were identified. Median age at diagnosis was 66 years (range: 34 - 95) and 97\% of the patients were female. Of all patients, 23 (64\%) had indolent lymphoma and 13 (36\%) had aggressive lymphoma. The most common histologic subtypes were marginal zone lymphoma (33\%), DLBCL (25\%), and follicular lymphoma (19\%). Only two (6\%) patients had implant-associated ALCL and one (3\%) patient had classic nodular sclerosing Hodgkin lymphoma. Five (14\%) patients had bilateral breast involvement at the time of diagnosis. Among all patients, 27 (75\%) had Stage IE, six (17\%) had Stage IIE, and three (8\%) had Stage IV disease at the time of diagnosis. Patients with indolent PBL were more often asymptomatic and diagnosed via screening mammograms compared to those with aggressive PBL (78\% vs. $31 \%, p=0.005$, respectively). On the contrary, patients with aggressive PBL more often presented with a breast lump and/or B-symptoms compared to those with indolent PBL (69\% vs. $22 \%, \mathrm{p}=0.005$, respectively). Only two (6\%) patients in the entire cohort presented with B symptoms, along with a breast lump, at the time of diagnosis. Table 1 summarizes the baseline and treatment characteristics of the patients.

\begin{tabular}{|c|c|c|c|c|}
\hline Characteristics & All Patients $\mathrm{n}(\%)$ & Indolent $\mathbf{n}(\%)$ & Aggressive $\mathrm{n}(\%)$ & P-value \\
\hline Age (year), median (range) & $66(34-95)$ & $66(34-83)$ & $67(49-95)$ & 0.46 \\
\hline Age $>65$ & 21 (58.3) & $12(52.2)$ & 9 (69.2) & 0.48 \\
\hline Male & $1(2.8)$ & $0(0)$ & $1(7.7)$ & 0.36 \\
\hline Female & $35(97.2)$ & $23(100)$ & $12(92.3)$ & 0.47 \\
\hline White ethnicity & 35 (97.2) & 22 (95.7) & $13(100)$ & 1 \\
\hline African American & $1(2.8)$ & $1(4.3)$ & $0(0)$ & 0.65 \\
\hline \multicolumn{5}{|l|}{ Clinical presentation at diagnosis } \\
\hline Abnormal screening mammogram & $22(61.1)$ & $18(78.3)$ & $4(30.8)$ & 0.005 \\
\hline Breast lump/swelling & $14(38.9)$ & $5(21.7)$ & $9(69.2)$ & 0.005 \\
\hline B-symptoms & $2(5.6)$ & $1(4.3)$ & $1(7.7)$ & 0.67 \\
\hline ECOG performance score at diagnosis & & & & 0.31 \\
\hline
\end{tabular}




\section{Cureus}

\begin{tabular}{|c|c|c|c|c|}
\hline 0 or 1 & 34 (94.4) & $22(95.7)$ & $12(92.3)$ & \\
\hline 2 & $1(2.8)$ & $1(4.3)$ & $0(0)$ & \\
\hline 3 & $1(2.8)$ & $0(0)$ & $1(7.7)$ & \\
\hline Laterality of tumor & & & & 0.69 \\
\hline Right & $14(38.9)$ & $9(39.1)$ & $5(38.5)$ & \\
\hline Left & $17(47.2)$ & $10(43.5)$ & $7(53.8)$ & \\
\hline Bilateral & $5(13.9)$ & $4(17.4)$ & $1(7.7)$ & \\
\hline Stage at diagnosis & & & & 0.74 \\
\hline IE & $27(75.0)$ & $18(78.3)$ & $9(69.2)$ & \\
\hline IIE & $6(16.7)$ & $3(13.0)$ & $3(23.1)$ & \\
\hline IV & $3(8.3)$ & $2(8.7)$ & $1(7.7)$ & \\
\hline Histologic type & & & & - \\
\hline Marginal zone & $12(33.3)$ & $12(52.2)$ & $0(0)$ & \\
\hline Diffuse large B cell lymphoma & $9(25.0)$ & $0(0)$ & $9(69.2)$ & \\
\hline Follicular lymphoma & $7(19.4)$ & $7(30.4)$ & $0(0)$ & \\
\hline CLL/SLL & $3(8.3)$ & $3(13.0)$ & $0(0)$ & \\
\hline BIA-ALCL & $2(5.6)$ & $0(0)$ & $2(15.4)$ & \\
\hline BCL-U (DLBCL or Burkitt lymphoma) & $2(5.6)$ & $0(0)$ & $2(15.4)$ & \\
\hline Nodular sclerosing Hodgkin lymphoma & $1(2.8)$ & $1(4.3)$ & $0(0)$ & \\
\hline \multicolumn{5}{|l|}{ Treatment types } \\
\hline Observation & $6(16.7)$ & $6(26.1)$ & $0(0)$ & 0.07 \\
\hline Chemotherapy only & $9(25.0)$ & $6(26.1)$ & $3(23.1)$ & 0.84 \\
\hline Radiotherapy only & $7(19.4)$ & $5(21.7)$ & $2(15.4)$ & 0.64 \\
\hline Surgery only & $2(5.6)$ & $1(4.3)$ & $1(7.7)$ & 1 \\
\hline Surgery/chemotherapy & $2(5.6)$ & $1(4.3)$ & $1(7.7)$ & 1 \\
\hline Chemotherapy/radiotherapy & $8(22.2)$ & $2(8.7)$ & $6(46.2)$ & 0.009 \\
\hline Surgery/radiotherapy & $1(2.8)$ & $1(4.3)$ & $0(0)$ & 1 \\
\hline Surgery/chemotherapy/radiotherapy & $1(2.8)$ & $1(4.3)$ & $0(0)$ & 1 \\
\hline
\end{tabular}

\section{TABLE 1: Patient and Treatment Characteristics}

BCL-U: B-cell lymphoma - unclassifiable; BIA-ALCL: breast implant-associated anaplastic large T-cell lymphoma; CLL/SLL: chronic lymphocytic leukemia/small lymphocytic leukemia; DLBCL: diffuse large B-cell lymphoma; ECOG: Eastern Cooperative Oncology Group

Commonly used treatment modalities for aggressive vs. indolent PBL were chemotherapy alone (23\% vs. $26 \%, p=0.8)$, chemoradiotherapy ( $46 \%$ vs. $9 \%, p=0.009)$, radiotherapy alone $(15 \%$ vs. $22 \%, p=0.6)$, and observation ( $0 \%$ vs. $26 \%, p=0.07)$, respectively. Commonly used chemotherapy regimens in aggressive vs. indolent PBL were rituximab, cyclophosphamide, doxorubicin hydrochloride, vincristine (Oncovin), and prednisone (R-CHOP) ( $40 \%$ vs. $40 \%, \mathrm{p}=1)$, rituximab alone $(10 \%$ vs. $30 \%, \mathrm{p}=0.58)$, rituximab, etoposide phosphate, prednisone, vincristine sulfate (Oncovin), cyclophosphamide, and doxorubicin hydrochloride (hydroxydaunorubicin) $(\mathrm{R}-\mathrm{EPOCH})(30 \%$ vs. $0 \%, \mathrm{p}=0.21)$, and rituximab/bendamustine $(0 \%$ vs. $20 \%, \mathrm{p}=$ 0.47 ), respectively (Table 2). Surgery was performed for six (17\%) patients; three underwent a lumpectomy, two underwent implant removal with complete capsulectomy for implant-associated ALCL, and one underwent a partial mastectomy. Single and a combination of more than one treatment modality were used for six $(46 \%)$ aggressive vs. $12(52 \%)$ indolent $(\mathrm{p}=0.7)$ and seven $(54 \%)$ aggressive vs. five $(22 \%)$ indolent $(\mathrm{p}=$ $0.05)$ PBL cases, respectively. Prophylactic intrathecal chemotherapy was used in one patient with Stage IIE 


\section{Cureus}

bilateral PBL and this patient didn’t have any central nervous system (CNS) relapse.

\begin{tabular}{|c|c|c|c|c|}
\hline Chemotherapy regimens & All Patients n (\%) & Indolent n (\%) & Aggressive n (\%) & P-value \\
\hline R-CHOP & $8(40)$ & $4(40)$ & $4(40)$ & 1 \\
\hline Rituximab & $4(20)$ & $3(30)$ & $1(10)$ & 0.58 \\
\hline R-EPOCH & $3(15)$ & $0(0)$ & $3(30)$ & 0.21 \\
\hline Rituximab/bendamustine & $2(10)$ & $2(20)$ & $0(0)$ & 0.47 \\
\hline ABVD & 1 (5) & $1(10)$ & $0(0)$ & 1 \\
\hline CHOEP & 1 (5) & $0(0)$ & $1(10)$ & 1 \\
\hline CODOX & 1 (5) & $0(0)$ & 1 (10) & 1 \\
\hline
\end{tabular}

\section{TABLE 2: First-Line Chemotherapy Regimens}

ABVD: Adriamycin (doxorubicin), bleomycin, vinblastine, dacarbazine; CHOEP: cyclophosphamide, doxorubicin, etoposide, vincristine, prednisone; CODOX: cyclophosphamide, vincristine, doxorubicin; R-CHOP: rituximab, cyclophosphamide, doxorubicin, vincristine, prednisone; R-EPOCH: rituximab, etoposide phosphate, prednisone, vincristine sulfate (Oncovin), cyclophosphamide, and doxorubicin hydrochloride (hydroxydaunorubicin)

The median follow-up of these patients was 37 months. Among six (17\%) patients who had disease relapse, only one patient with Stage IE primary breast DLBCL had CNS relapse, and another patient died. Six (17\%) patients died, of which all were above 65 years old at the time of diagnosis; three were observed for indolent PBL, one received radiotherapy for indolent PBL, and two patients received radiotherapy alone and chemoradiotherapy for aggressive PBL. The five-year OS and PFS of PBL patients were 82\% (95\% CI: 67 $100)$ and 63\% (95\% CI: $45-89$ ), respectively. Five-year OS and PFS of patients with aggressive vs. indolent PBL were $92 \%$ (95\% CI: 77 - 100) vs. 80\% (95\% CI: 63 - 100), p = 0.6, and $84 \%$ (95\% CI: 66 - 100) vs. $60 \%$ (95\% CI: $39-91$ ), $p=0.9$, respectively (Figure $1 A-B$ ). The five-year OS of patients who received a combination of more than one treatment modality for PBL was 92\% (95\% CI: 77 - 100) compared to 86\% (95\% CI: 63 - 100), p $=0.9$ and $53 \%$ (95\% CI: $21-100), p=0.07$ for patients who were treated with a single treatment modality and observed, respectively (Figure 1C). 


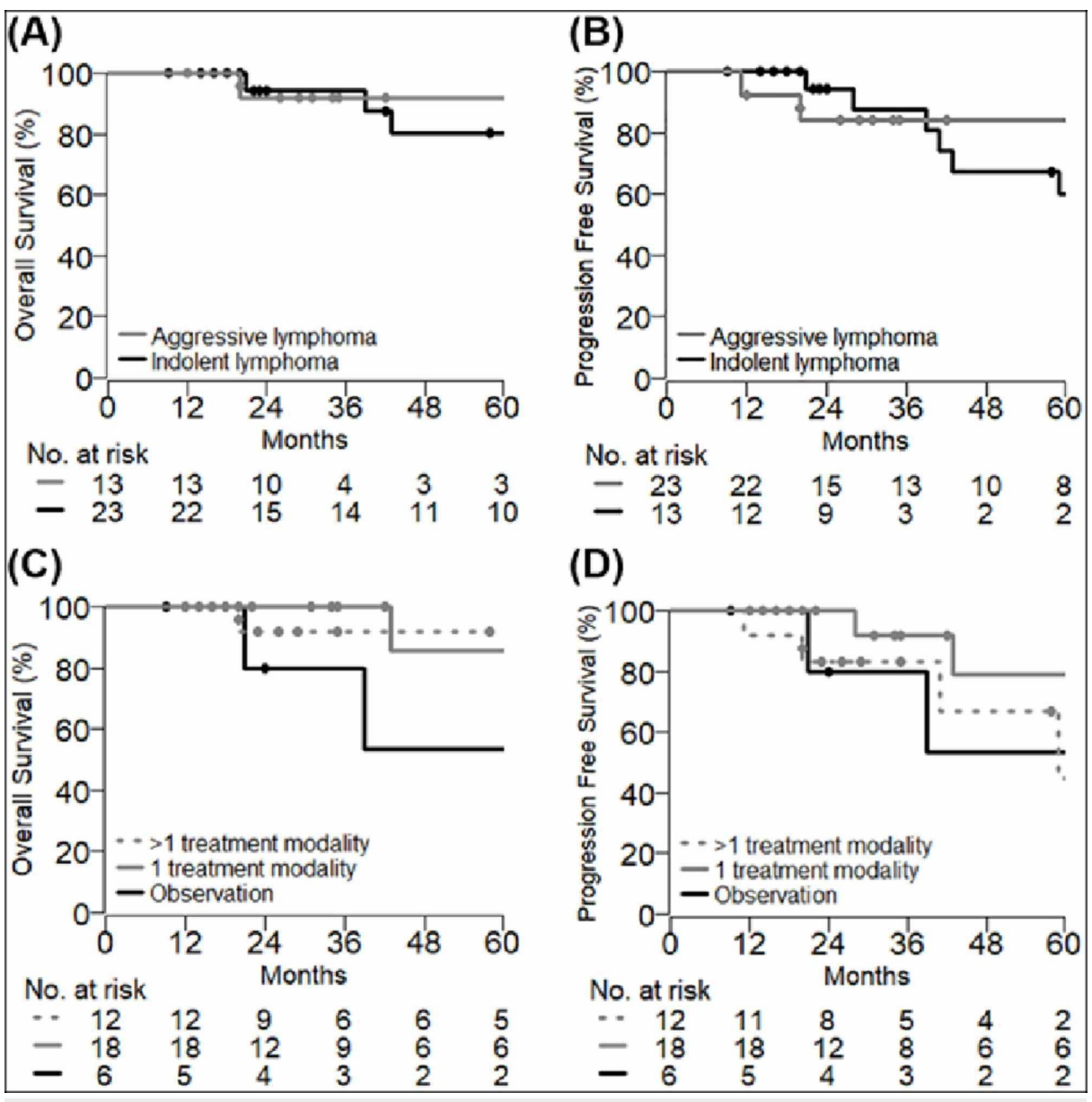

FIGURE 1: Overall survival and relapse-free survival of patients with primary breast lymphoma

(A - B) Stratified based on histologic subtype; (C - D) Stratified based on number of treatment modalities

\section{Discussion}

The typical clinical presentation of PBL is with a solitary breast lump in female patients between 50 and 70 years of age $[1,8]$. Similarly, $39 \%$ of the patients presented with a breast lump, and the median age of the patients was 66 years in our study. Although DLBCL was the most common histological subtype in previous studies, marginal zone lymphoma was the most common histological subtype in our cohort and only $25 \%$ of our patients had DLBCL $[1,3,5-6]$. The five-year OS of PBL patients in the present study was similar to what has been previously reported $[1,9,19,21]$. Tumor size, laterality, and histological lymphoma subtype were not prognostic indicators in our study and the majority of the prior studies [1, 4, 6-9, 21]. Although Zu et al. reported Ann Arbor's clinical stage and B symptoms as independent prognostic factors for OS and PFS, they were not predictors of OS or PFS in our cohort [9].

In a retrospective study by Ryan et al., treatment with anthracycline-containing chemotherapy and radiotherapy prolonged the OS [19]. Prior studies showed that radiotherapy for Stage I and chemotherapy for Stage II PBL patients prolonged both OS and PFS [22-23]. To date, only one randomized clinical trial was performed for PBL patients in which chemoradiotherapy prolonged OS and event-free survival more than chemotherapy or radiotherapy alone [24]. In our cohort, aggressive PBL cases were more often treated with chemoradiotherapy compared to indolent PBL cases. Importantly, three of six (50\%) patients with indolent PBL who were observed without any treatment died within four years of diagnosis. This finding suggests that the use of at least a single treatment modality should be considered, even for patients with indolent PBL. However, there was no statistically significant difference between the different treatment modalities, maybe in part due to the important limitations of our study, such as the single-center retrospective study design with a small number of patients. 
use of rituximab and radiotherapy in primary breast DLBCL has been found to improve OS and reduce relapse rates $[3,16,18]$. On the other hand, Hosein et al. noted in a retrospective study that rituximab use was not associated with any survival benefit [21]. To date, no randomized clinical trials were performed to assess the efficacy of rituximab for the treatment of PBL. In the present study, $85 \%$ of the patients treated with chemotherapy and $47 \%$ of the whole cohort received rituximab.

The overall rate of CNS relapse in patients with PBL has been reported to be around 8\% [24]. Prophylactic intrathecal chemotherapy is controversial and should only be considered in high-risk patients, such as patients with Stage IIE disease and aggressive lymphoma histology [1, 13, 22]. CNS relapse was less common in our cohort with a rate of $3 \%$.

\section{Conclusions}

In this cohort of patients with PBL, marginal zone lymphoma was the most common histological subtype and mortality was seen only in elderly patients regardless of the histologic subtype. Higher utilization of chemoradiotherapy in aggressive PBL was able to overcome the worse prognosis of these patients. At least one treatment modality should be considered in patients with indolent PBL given that observation alone was associated with a poor prognosis.

\section{Additional Information \\ Disclosures}

Human subjects: Consent was obtained by all participants in this study. Institutional Review Board of the Cleveland Clinic issued approval 18-1303. Animal subjects: All authors have confirmed that this study did not involve animal subjects or tissue. Conflicts of interest: In compliance with the ICMJE uniform disclosure form, all authors declare the following: Payment/services info: All authors have declared that no financial support was received from any organization for the submitted work. Financial relationships: All authors have declared that they have no financial relationships at present or within the previous three years with any organizations that might have an interest in the submitted work. Other relationships: All authors have declared that there are no other relationships or activities that could appear to have influenced the submitted work.

\section{References}

1. Franco Pérez F, Lavernia J, Aguiar-Bujanda D, et al.: Primary breast lymphoma: analysis of 55 cases of the Spanish lymphoma oncology group. Clin Lymphoma Myeloma Leuk. 2017, 17:186-191. 10.1016/j.clml.2016.09.004

2. Thomas A, Link BK, Altekruse S, Romitti PA, Schroeder MC: Primary breast lymphoma in the United States: 1975-2013. J Natl Cancer Inst. 2017, 109:djw294. 10.1093/jnci/djw294

3. Jia Y, Sun C, Liu Z, Wang W, Zhou X: Primary breast diffuse large B-cell lymphoma: a population-based study from 1975 to 2014. Oncotarget. 2017, 9:3956-3967. 10.18632/oncotarget.23285

4. Wiseman C, Liao KT: Primary lymphoma of the breast. Cancer. 1972, 29:1705-1712. 10.1002/10970142(197206)29:6<1705::aid-cncr2820290640>3.0.co;2-i

5. Hugh JC, Jackson FI, Hanson J, Poppema S: Primary breast lymphoma: an immunohistologic study of 20 new cases. Cancer. 1990, 66:2602-2611. 10.1002/1097-0142(19901215)66:12<2602::aidcncr2820661224>3.0.co;2-u

6. Cheah CY, Campbell BA, Seymour JF: Primary breast lymphoma. Cancer Treat Rev. 2014, 40:900-908. 10.1016/j.ctrv.2014.05.010

7. Shen H, Wei Z, Zhou D, et al.: Primary extra-nodal diffuse large B-cell lymphoma: a prognostic analysis of 141 patients. Oncol Lett. 2018, 16:1602-1614. 10.3892/ol.2018.8803

8. Raj SD, Shurafa M, Shah Z, Raj KM, Fishman MDC, Dialani VM: Primary and secondary breast lymphoma: clinical, pathologic, and multimodality imaging review. Radiographics. 2019, 39:610-625. 10.1148/rg.2019180097

9. Zhu YH, Meng WJ, He LH, Jia YS, Tong ZS: Prognostic analysis of primary breast diffuse large B cell lymphoma (Article in Chinese). Zhonghua Zhong Liu Za Zhi. 2019, 41:235-240.

10. Zhang N, Cao C, Zhu Y, et al.: Primary breast lymphoma: a single center study . Oncol Lett. 2017, 13:10141018. 10.3892/ol.2016.5483

11. Ludmir EB, Milgrom SA, Pinnix CC, et al.: Emerging treatment strategies for primary breast extranodal marginal zone lymphoma of mucosa-associated lymphoid tissue. Clin Lymphoma Myeloma Leuk. 2019, 19:244-250. 10.1016/j.clml.2018.12.016

12. Gualco G, Chioato L, Harrington WJ Jr, Weiss LM, Bacchi CE: Primary and secondary T-cell lymphomas of the breast: clinico-pathologic features of 11 cases. Appl Immunohistochem Mol Morphol. 2009, 17:301-306. 10.1097/PAI.0b013e318195286d

13. Validire $\mathrm{P}$, Capovilla M, Asselain B, et al.: Primary breast non-Hodgkin's lymphoma: a large single center study of initial characteristics, natural history, and prognostic factors. Am J Hematol. 2009, 84:133-139. 10.1002/ajh.21353

14. Yhim HY, Kang HJ, Choi YH, et al.: Clinical outcomes and prognostic factors in patients with breast diffuse large B cell lymphoma; Consortium for Improving Survival of Lymphoma (CISL) study. BMC Cancer. 2010, 10:321. 10.1186/1471-2407-10-321

15. Dong LH, Chen JL, Gao X, Li GP, Li YF, Song YP, Wei XD: Clinical efficacy of EPOCH+/-R followed by DICE+/-R regimen for primary breast diffuse large B-cell lymphoma (Article in Chinese). Zhongguo Shi Yan 


\section{Cureus}

Xue Ye Xue Za. 2017, 25:766-771

16. Hu S, Song Y, Sun X, et al.: Primary breast diffuse large B-cell lymphoma in the rituximab era: therapeutic strategies and patterns of failure. Cancer Sci. 2018, 109:3943-3952. 10.1111/cas.13828

17. Ludmir EB, Milgrom SA, Pinnix CC, et al.: Primary breast diffuse large B-cell lymphoma: treatment strategies and patterns of failure. Leuk Lymphoma. 2018, 59:2896-2903. 10.1080/10428194.2018.1460825

18. Liu PP, Wang KF, Jin JT, et al.: Role of radiation therapy in primary breast diffuse large B-cell lymphoma in the Rituximab era: a SEER database analysis. Cancer Med. 2018, 7:1845-1851. 10.1002/cam4.1457

19. Ryan G, Martinelli G, Kuper-Hommel M, et al.: Primary diffuse large B-cell lymphoma of the breast: prognostic factors and outcomes of a study by the International Extranodal Lymphoma Study Group. Ann Oncol. 2008, 19:233-241. 10.1093/annonc/mdm471

20. Harris NL, Jaffe ES, Diebold J, et al.: The World Health Organization classification of neoplastic diseases of the haematopoietic and lymphoid tissues: report of the Clinical Advisory Committee Meeting, Airlie House, Virginia, November 1997. Histopathology. 2000, 36:69-86. 10.1046/j.1365-2559.2000.00895.x

21. Hosein PJ, Maragulia JC, Salzberg MP, et al.: A multicentre study of primary breast diffuse large B-cell lymphoma in the rituximab era. Br J Haematol. 2014, 165:358-363. 10.1111/bjh.12753

22. Jennings WC, Baker RS, Murray SS, et al.: Primary breast lymphoma: the role of mastectomy and the importance of lymph node status. Ann Surg. 2007, 245:784-789. 10.1097/01.sla.0000254418.90192.59

23. Zhao S, Zhang QY, Ma WJ, et al.: Analysis of 31 cases of primary breast lymphoma: the effect of nodal involvement and microvascular density. Clin Lymphoma Myeloma Leuk. 2011, 11:33-37. 10.3816/CLML.2011.n.004

24. Avilés A, Delgado S, Nambo MJ, Neri N, Murillo E, Cleto S: Primary breast lymphoma: results of a controlled clinical trial. Oncology. 2005, 69:256-260. 10.1159/000088333

25. Aviv A, Tadmor T, Polliack A: Primary diffuse large B-cell lymphoma of the breast: looking at pathogenesis, clinical issues and therapeutic options. Ann Oncol. 2013, 24:2236-2244. 10.1093/annonc/mdt192 